\title{
Article \\ Hidden Pseudogap and Excitation Spectra in a Strongly Coupled Two-Band Superfluid/Superconductor
}

\author{
Hiroyuki Tajima $1, * \mathbb{D}$, Pierbiagio Pieri $2,3, * \mathbb{1}$ and Andrea Perali $4, * \mathbb{( D}$ \\ 1 Department of Mathematics and Physics, Kochi University, Kochi 780-8520, Japan \\ 2 Dipartimento di Fisica e Astronomia, Università di Bologna, 40127 Bologna, Italy \\ 3 INFN, Sezione di Bologna, 40127 Bologna, Italy \\ 4 Physics Unit, School of Pharmacy, Università di Camerino, 62032 Camerino, Italy \\ * Correspondence: htajima@kochi-u.ac.jp (H.T.); pierbiagio.pieri@unibo.it (P.P.); andrea.perali@unicam.it (A.P.)
}

check for

updates

Citation: Tajima, H.; Pieri, P.; Perali, A. Hidden Pseudogap and Excitation Spectra in a Strongly Coupled TwoBand Superfluid/Superconductor. Condens. Matter 2021, 6, 8. https:// doi.org/10.3390/condmat6010008

Received: 23 December 2020 Accepted: 3 February 2021 Published: 7 February 2021

Publisher's Note: MDPI stays neutral with regard to jurisdictional clai$\mathrm{ms}$ in published maps and institutional affiliations.

Copyright: $\odot 2021$ by the authors. Licensee MDPI, Basel, Switzerland. This article is an open access article distributed under the terms and conditions of the Creative Commons Attribution (CC BY) license (https:// creativecommons.org/licenses/by/ $4.0 /)$.

\begin{abstract}
We investigate single-particle excitation properties in the normal state of a two-band superconductor or superfluid throughout the Bardeen-Cooper-Schrieffer (BCS) to Bose-Einsteincondensation (BEC) crossover, within the many-body $T$-matrix approximation for multichannel pairing fluctuations. We address the single-particle density of states and the spectral functions consisting of two contributions associated with a weakly interacting deep band and a strongly interacting shallow band, relevant for iron-based multiband superconductors and multicomponent fermionic superfluids. We show how the pseudogap state in the shallow band is hidden by the deep band contribution throughout the two-band BCS-BEC crossover. Our results could explain the missing pseudogap in recent scanning tunneling microscopy experiments in FeSe superconductors.
\end{abstract}

Keywords: BCS-BEC crossover; pseudogap; spectral function; multiband superconductor; multicomponent superfluids

\section{Introduction}

Recently, the Bardeen-Cooper-Schrieffer to Bose-Einstein-condensation (BCS-BEC) crossover, where a weakly-interacting BCS state continuously changes to BEC of tightly bound molecules with increasing the attractive interaction [1-4], has gathered much attention due to its realization in ultracold Fermi gases [5,6]. Moreover, such a crossover phenomenon has been confirmed experimentally in multiband iron-based superconductors, such as the iron-chalcogenides family FeSe [7-11].

Thanks to these experimental progress, the two-band BCS-BEC crossover theory has been of particular interest in condensed matter and ultracold atomic physics [12-22]. In particular, the FeSe superconductors involve a multiband configuration which plays a crucial role for the BCS-BEC crossover. Moreover, the most fundamental two-band model for multichannel pairing proposed by Suhl, Matthias and Walker [23] has been realized in $\mathrm{Yb}$ ultracold Fermi gases near an orbital Feshbach resonance [24-26].

One of the exciting topics in the two-band BCS-BEC crossover is the existence of pseudogaps in the single-particle excitation in the normal state (for reviews discussing the pseudogap, see [27-29]). While several experiments for FeSe report signals of pseudogaps and preformed Cooper pairs [30-33], a recent scanning tunneling spectroscopy (STS) measurement did not observe a pseudogap behavior even in the crossover regime of the BCS-BEC crossover [34]. In addition, a torque magnetometry experiment in the same system indicates weak pairing fluctuations [35]. Theoretically, the screening of pairing fluctuations originating from the two-band configuration with different pairing strengths has been reported [36-40], but the perfect screening observed in the experiment [34] may require a further mechanism for suppressing the pseudogap.

In this article, we resolve this complicated phenomenology by calculating the singleparticle density of states and spectral function throughout the two-band BCS-BEC crossover. 
We adopt the many-body T-matrix approximation (TMA) for multichannel pairing fluctuations in the normal state. We show that the pseudogap occurring in the strongly coupled shallow band is masked by the contribution from the deep band in the total (i.e., summed over the bands) density of state, which is measured in the STS experiment. This masking effect becomes remarkable in the strong-coupling regime for the shallow band due to the overlap of spectral weights in each band. On the other hand, we show that the total spectral function relevant for the angular-resolved-photoemission spectroscopy (ARPES) clearly reflects the pseudogap features in the strongly coupled shallow band.

\section{Formalism}

We consider a three-dimensional two-band model for attractive fermions described by

$$
H=\sum_{\mathbf{k}, \sigma, j} \xi_{\mathbf{k} j} c_{\mathbf{k} \sigma j}^{\dagger} c_{\mathbf{k} \sigma j}+\sum_{j_{1}, j_{2}} \sum_{\mathbf{q}} V_{j_{1} j_{2}} b_{\mathbf{q} j_{1}}^{\dagger} b_{\mathbf{q} j_{2}}
$$

where $c_{\mathbf{k} \sigma j}$ is the annihilation operator of a fermion with the momentum $\mathbf{k}$, spin $\sigma=\uparrow, \downarrow$ and the band index $j$ (where $j=1$ and $j=2$ denote the indices of deep and shallow bands, respectively) and $\xi_{\mathbf{k} j}=k^{2} /\left(2 m_{j}\right)-\mu+E_{0} \delta_{j, 2}$ is the single-particle dispersion in the $j$-band, measured from the chemical potential $\mu$ with the energy separation $E_{0}$ between the two bands. For simplicity, we take the same effective mass $m=m_{1}=m_{2}$ in each band. Hereafter, we take $\hbar=k_{\mathrm{B}}=1$ and the unit volume. We define a pair-annihilation operator

$$
b_{\mathbf{q} j}=\sum_{\mathbf{k}} c_{-\mathbf{k}+\mathbf{q} / 2 \downarrow j} c_{\mathbf{k}+\mathbf{q} / 2 \uparrow j} .
$$

We employ a contact-type interaction. In Equation (1), the second term represents the pair-scattering process between $j_{1}$ and $j_{2}$ bands. Specifically, the intraband couplings $V_{11}$ and $V_{22}$ are expressed in terms of the corresponding scattering lengths $a_{11}$ and $a_{22}$ as [13]

$$
\frac{m}{4 \pi a_{j j}}=\frac{1}{V_{j j}}+\sum_{\mathbf{k}}^{|\mathbf{k}| \leq k_{0}} \frac{m}{k^{2}}
$$

where the momentum cutoff $k_{0}$ is taken much larger than all other momentum scales.

Superconducting pair-fluctuation effects are incorporated by the two-channel $T$ matrix $[39,40]$

$$
\begin{aligned}
\left(\begin{array}{ll}
T_{11}\left(\mathbf{q}, i v_{\ell}\right) & T_{12}\left(\mathbf{q}, i v_{\ell}\right) \\
T_{21}\left(\mathbf{q}, i v_{\ell}\right) & T_{22}\left(\mathbf{q}, i v_{\ell}\right)
\end{array}\right) & =\left(\begin{array}{ll}
V_{11} & V_{12} \\
V_{21} & V_{22}
\end{array}\right)+\left(\begin{array}{ll}
V_{11} & V_{12} \\
V_{21} & V_{22}
\end{array}\right)\left(\begin{array}{cc}
\chi_{11}\left(\mathbf{q}, i v_{\ell}\right) & 0 \\
0 & \chi_{22}\left(\mathbf{q}, i v_{\ell}\right)
\end{array}\right) \\
& \times\left(\begin{array}{ll}
T_{11}\left(\mathbf{q}, i v_{\ell}\right) & T_{12}\left(\mathbf{q}, i v_{\ell}\right) \\
T_{21}\left(\mathbf{q}, i v_{\ell}\right) & T_{22}\left(\mathbf{q}, i v_{\ell}\right)
\end{array}\right),
\end{aligned}
$$

where

$$
\chi_{j j}\left(\mathbf{q}, i v_{\ell}\right)=\sum_{\mathbf{k}} \frac{1-f\left(\xi_{\mathbf{k}+\mathbf{q} / 2 \uparrow j}\right)-f\left(\xi_{-\mathbf{k}+\mathbf{q} / 2 \downarrow j}\right)}{i v_{\ell}-\xi_{\mathbf{k}+\mathbf{q} / 2 \uparrow j}-\xi_{-\mathbf{k}+\mathbf{q} / 2 \downarrow j}}
$$

is the lowest order particle-particle correlation function in the $j$-band. $v_{\ell}=2 \ell \pi T$ is the bosonic Matsubara frequency. In Equation (5), $f(x)=\left(e^{x / T}+1\right)^{-1}$ is the Fermi-Dirac distribution function. The diagonal components read

$$
T_{j j}\left(\mathbf{q}, i v_{\ell}\right)=\frac{V_{j j}\left[1-V_{\overline{j j}} \chi_{j j}\left(\mathbf{q}, i v_{\ell}\right)\right]+V_{12} V_{21} \chi_{\overline{j j}}\left(\mathbf{q}, i v_{\ell}\right)}{\left[1-V_{j j} \chi_{j j}\left(\mathbf{q}, i v_{\ell}\right)\right]\left[1-V_{\overline{j j}} \chi_{\overline{j j}}\left(\mathbf{q}, i v_{\ell}\right)\right]-V_{12} V_{21} \chi_{\overline{j j}}\left(\mathbf{q}, i v_{\ell}\right) \chi_{j j}\left(\mathbf{q}, i v_{\ell}\right)}
$$


where $\bar{j}$ denotes the other band with respect to band $j$. In the two-channel $T$-matrix approach, the fermionic self-energy is of the form

$$
\Sigma_{j}\left(\mathbf{k}, i \omega_{s}\right)=T \sum_{\mathbf{q}} \sum_{\ell} T_{j j}\left(\mathbf{q}, i v_{\ell}\right) G_{j}^{(0)}\left(\mathbf{q}-\mathbf{k}, i v_{\ell}-i \omega_{n}\right),
$$

where $G_{j}^{(0)}\left(\mathbf{k}, i \omega_{s}\right)=\left(i \omega_{s}-\xi_{\mathbf{k} j}\right)^{-1}$ is the bare electron propagator with the fermionic Matsubara frequency $\omega_{s}=(2 s+1) \pi T$. We note that the off-diagonal components of the two-band $T$-matrix $T_{12}\left(\mathbf{q}, i v_{\ell}\right)$ and $T_{21}\left(\mathbf{q}, i v_{\ell}\right)$ are not involved in the self-energy since the band indices are conserved in the external lines. The dressed propagator $G_{j}\left(\mathbf{k}, i \omega_{s}\right)$ obeys the Dyson's equation

$$
G_{j}\left(\mathbf{k}, i \omega_{s}\right)=G_{j}^{(0)}\left(\mathbf{k}, i \omega_{s}\right)+G_{j}^{(0)}\left(\mathbf{k}, i \omega_{s}\right) \Sigma_{j}\left(\mathbf{k}, i \omega_{s}\right) G_{j}\left(\mathbf{k}, i \omega_{s}\right) .
$$

In the absence of $\Sigma_{j}\left(\mathbf{k}, i \omega_{s}\right)$, the single-particle spectrum is free-fermion-like and exhibits two quadratic bands. On the other hand, this self-energy correction leads to the pseudogap opening around the Fermi energy [40].

In the STS experiment, one observes the tunneling current $I$ occurring via the tunneling Hamiltonian [41]

$$
H_{\mathrm{T}}=\sum_{j} \sum_{\mathbf{k}, \mathbf{k}^{\prime}} \sum_{\sigma, \sigma^{\prime}}\left[t_{j} c_{\mathbf{k} \sigma j}^{\dagger} c_{\mathbf{k}^{\prime} \sigma^{\prime} 0}+\text { h.c. }\right]
$$

where $c_{\mathbf{k}^{\prime} \sigma^{\prime} 0}$ denotes the annihilation operator of an electron in the weakly-coupled normal metal connected to the sample. For simplicity, we consider the momentum-, spin-, and band-independent tunneling amplitudes $t=t_{1}=t_{2}$. The tunneling current is obtained as

$$
I=2 \pi e \int d \omega[f(\omega-e V)-f(\omega)] \sum_{j} \sum_{\mathbf{k}, \mathbf{k}^{\prime}}|t|^{2} A_{j}(\mathbf{k}, \omega-e V) A_{\mathrm{r}}\left(\mathbf{k}^{\prime}, \omega\right),
$$

where $V$ is the bias voltage and $e$ is an electron charge. In Equation $(10), A_{j}(\mathbf{k}, \omega)$ is the spectral function given by

$$
A_{j}(\mathbf{k}, \omega)=-\frac{1}{\pi} \operatorname{Im} G_{j}\left(\mathbf{k}, i \omega_{s} \rightarrow \omega+i \delta\right),
$$

where $\delta$ is an infinitesimal small positive number to generate the retarded Green's function for real frequencies. $A_{\mathrm{r}}\left(\mathbf{k}^{\prime}, \omega\right)$ is the spectral function in the reference normal metal. At sufficiently low temperature, we obtain the differential conductance

$$
\frac{d I}{d V}=2 \pi e|t|^{2} N_{\mathrm{r}}(0) N(e V),
$$

where

$$
N(\omega)=\sum_{j} \sum_{\mathbf{k}} A_{j}(\mathbf{k}, \omega)
$$

is the total density of states. $N_{r}(0)$ is the density of states at the Fermi level in the reference metal in the normal state. In this way, one can observe if a pseudogap opens in the total density of states.

In this article, we examine $N(\omega)$ at the superconducting (or superfluid) critical temperature $T_{\mathrm{c}}$ identified by the Thouless criterion given by

$$
\left[1+V_{11} \chi_{11}(\mathbf{0}, 0)\right]\left[1+V_{22} \chi_{22}(\mathbf{0}, 0)\right]-V_{12} V_{21} \chi_{11}(\mathbf{0}, 0) \chi_{22}(\mathbf{0}, 0)=0 .
$$


The chemical potential $\mu$ is determined by the density equation

$$
n=2 T \sum_{j} \sum_{\mathbf{k}} \sum_{s} G_{j}\left(\mathbf{k}, i \omega_{s}\right) .
$$

Note that one obtains $n=n_{1}+n_{2}$ with $n_{j}=\frac{k_{\mathrm{F}, j}^{3}}{3 \pi^{2}}$ in the absence of interactions at $T=0$, where $k_{\mathrm{F}, 1}=\sqrt{2 m E_{\mathrm{F}, 1}}$ and $k_{\mathrm{F}, 2}=\sqrt{2 m E_{\mathrm{F}, 2}} \equiv \sqrt{2 m\left(E_{\mathrm{F}, 1}-E_{0}\right)}$ are the band Fermi momenta $\left(E_{\mathrm{F}, j}\right.$ is the band Fermi energy). We take $E_{0}=\frac{3}{5} E_{\mathrm{F}, 1}$ such that the two deep and shallow (occupied) bands are overlapped. We choose the dimensionless coupling parameter in the deep band in the weak-coupling regime as $\left(k_{\mathrm{F}, 1} a_{11}\right)^{-1}=-2$, while the coupling parameter in the shallow band $\left(k_{\mathrm{F}, 2} a_{22}\right)^{-1}$ is tuned throughout the BCS-BEC crossover $\left(-1 \lesssim\left(k_{\mathrm{F}, 2} a_{22}\right)^{-1} \lesssim 1\right)$. We note that our two-channel $T$-matrix formalism can be applied also in the case of strong intraband coupling in the deep band. The dimensionless interband pair-exchange coupling is given by $\tilde{V}_{12}=\tilde{V}_{21}=U_{12}\left(k_{0} / k_{\mathrm{F}}\right)^{2} n / E_{\mathrm{F}}$ where $k_{\mathrm{F}}=(3 \pi n)^{1 / 3}$ and $E_{\mathrm{F}}=k_{\mathrm{F}}^{2} /(2 m)$ are the Fermi wave-vector and the Fermi energy for the total density $n$. We take $k_{0}=100 k_{\mathrm{F}}$ which is a sufficiently large wave-vector cutoff compared to all other momentum scales.

It should be noted that in this work we have considered the simplest isotropic dispersions of two electron-type bands with same effective masses, while realistic compensated semimetals FeSe contain more complicated electronic band structures and effective pairing interactions, including cross-band pairing terms in competition with intraband pairings. A mean-field analysis of the interplay between cross-band and intraband pairing, with applications to iron-based superconductors, has been recently reported in Ref. [42], while the effects of fluctuations and pseudogaps in this complex configuration is still an open and computational demanding problem. However, the shallow Fermi surface pocket coupled to the deep band in these materials, corresponding to the band 2 in this paper, indeed plays a significant role for the realization of the two-band BCS-BEC crossover in FeSe superconductors [7]. We only retain this two-band configuration with isotropic wave-vector dispersions to focus on pairing fluctuation effects along the BCS-BEC crossover. Indeed, the isotropic and quadratic band dispersion was employed in Ref. [10] to understand qualitative features of the BCS-BEC crossover in $\mathrm{Fe}_{1+y} \mathrm{Se}_{x} \mathrm{Te}_{1-x}$. In addition, this isotropic two-band system is equivalent to the model describing $\mathrm{Yb}$ Fermi gases with an orbital Feshbach resonance [24], allowing to trace conclusions on the two-band BCS-BEC crossover having universal character, of interest for multicomponent ultracold atomic superfluids and solid state superconductors.

\section{Results}

First, in Figure 1 we show the evolution of the critical temperature $T_{\mathrm{c}}$ across the BCSBEC crossover with increasing $\left(k_{\mathrm{F}, 2} a_{22}\right)^{-1}$ for three cases $\tilde{V}_{12}=0, \tilde{V}_{12}=1$, and $\tilde{V}_{12}=2$. In the weak-coupling BCS side $\left(k_{\mathrm{F}, 2} a_{22}\right)^{-1} \lesssim 0, T_{\mathrm{C}}$ exponentially increases as $\sim \exp \left(\frac{\pi}{2 k_{\mathrm{F}, 2} a_{22}}\right)$. The finite pair-exchange coupling $\tilde{V}_{12}$ gives an enhancement of $T_{\mathrm{c}}$. In the strong-coupling $\operatorname{BEC}$ side $\left(k_{\mathrm{F}, 2} a_{22}\right)^{-1} \gtrsim 0, T_{\mathrm{c}}$ approaches the Bose-Einstein condensation temperature $T_{\mathrm{BEC}}$ of tightly bound molecules given by $[28,29]$

$$
\begin{aligned}
T_{\mathrm{BEC}} & =\frac{\pi}{m}\left(\frac{n}{\zeta(3 / 2)}\right)^{\frac{2}{3}} \\
& \simeq 0.218 T_{\mathrm{F}}
\end{aligned}
$$

where $\zeta(3 / 2) \simeq 2.612$ is the Riemann zeta function. This indicates that all the particles in both bands form molecular condensates in the strong-coupling limit. Although the nonzero pair-exchange coupling $\tilde{V}_{12}$ does not give qualitative effects on $T_{\mathrm{c}}$ in this regime $\left[\left(k_{\mathrm{F}, 2} a_{22}\right)^{-1} \gtrsim 1\right]$, the coexistence of large Cooper pairs and small molecules has been discussed within the mean-field [21], NSR [38], and TMA [39,40] approaches. With increasing 
$V_{12}$, the two-band system undergoes the BCS-BEC crossover even in the case of weak intraband couplings $\left[\left(k_{\mathrm{F}, 2} a_{22}\right)^{-1} \lesssim 1\right]$. In this way, one can find that the BCS-BEC crossover is realized by increasing the interaction strength in the shallow band $\left(k_{\mathrm{F}, 2} a_{22}\right)^{-1}$ in the present two-band model.

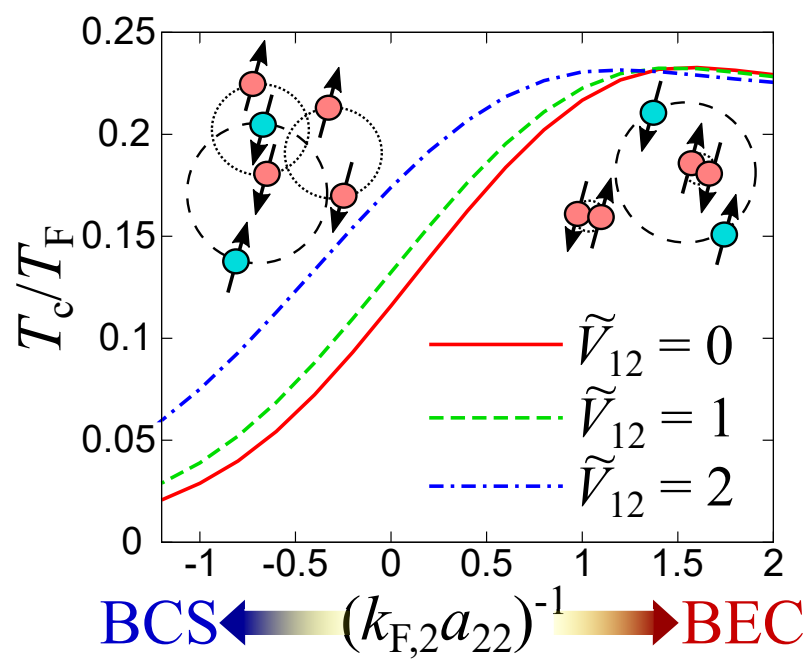

Figure 1. The superconducting (superfluid) critical temperature $T_{\mathrm{C}}$ throughout the two-band BCSBEC crossover at $\left(k_{\mathrm{F}, 1} a_{11}\right)^{-1}=-2$ with two cases of vanishing and finite pair-exchange couplings $\tilde{V}_{12}=0, \tilde{V}_{12}=1$, and $\tilde{V}_{12}=2 . T_{\mathrm{F}}=k_{\mathrm{F}}^{2} /(2 \mathrm{~m})$ is the Fermi temperature for the total number density $n$. A pictorial sketch of the two kind of Cooper pairs is given for illustration in the top part of the figure: the Cooper pairs in the shallow band (light red circles) undergo the BCS-BEC crossover, while Cooper pairs in the deep band (light blue circles) remains large, in the BCS regime.

In Figure 2a we show the evolution of the total density of states $N(\omega)$ throughout the two-band BCS-BEC crossover with vanishing pair-exchange coupling at $T=T_{\mathrm{c}}$. In the weak-coupling side for the shallow band (e.g., $\left(k_{\mathrm{F}, 2} a_{22}\right)^{-1} \leq-0.4$ in Figure 2a), one can see the pseudogap around the Fermi level $\omega=0$, which is small but with a relatively sharp dip structure. On the other hand, at unitarity (crossover regime, $\left(k_{\mathrm{F}, 2} a_{22}\right)^{-1}=0$ ) in the shallow band, the pseudogap is somehow hidden. Moreover, in the strong-coupling side of the BCS-BEC crossover (e.g., $\left(k_{\mathrm{F}, 2} a_{22}\right)^{-1} \geq 0.4$ in Figure $\left.2 \mathrm{a}\right), N(\omega)$ shows a nonmonotonic structure but not the fully gapped density of states which can be found in the single-band counterpart. To understand these behaviors, we examine the band-selective density of states given by

$$
N_{j}(\omega)=\sum_{\mathbf{k}} A_{j}(\mathbf{k}, \omega)
$$

In the inset of Figure 2, $N_{j}(\omega)$ for each band is plotted at $\left(k_{\mathrm{F}, 2} a_{22}\right)^{-1}=0$, corresponding to the crossover regime in the shallow band. $N_{2}(\omega)$ clearly exhibits the pseudogap behavior (dip structure around $\omega=0)$ in the shallow band $(j=2)$ due to the strong pairing fluctuations associated with $V_{22}$. However, the deep band $(j=1)$ shows the square-root behavior $N_{1}(\omega) \propto \sqrt{(\omega+\mu)}$ without the pseudogap signature in the case of $\tilde{V}_{12}=0$ because the intraband coupling is kept weak. In this regard, the pseudogap structure in the total $N(\omega)$ originating from $N_{2}(\omega)$ is hidden by the square-root contribution of $N_{1}(\omega)$. Such a situation occurs for larger intraband coupling in the shallow band (e.g., $\left(k_{\mathrm{F}, 2} a_{22}\right)^{-1}=0.4$ and 0.8 in Figure 2a). On the other hand, in the case of a finite interband pair-exchange coupling $\tilde{V}_{12}=1$, shown in Figure $2 \mathrm{~b}$, one can find a small pseudogap around $\omega=0$ in $N(\omega)$ even in the strong-coupling regime. Furthermore, $N(\omega)$ exhibits a large flattened region around the Fermi level $(\omega=0)$. These features can also be understood from the partial density of states $N_{i}(\omega)$ as shown in the inset for $\left(k_{\mathrm{F}, 2} a_{22}\right)^{-1}=0$. The pair-exchange process associated with finite $V_{12}$ induces the pseudogap even in the weakly interacting 
deep band $(j=1)$. Hence, one can find two pseudogaps with different sizes in the two bands. The resulting total density of states $N(\omega)$ exhibits the small pseudogap originating from $N_{1}(\omega)$ throughout the BCS-BEC crossover. On the other hand, the large pseudogap in $N_{2}(\omega)$ is hidden by the contribution of the sizable spectral weight of $N_{1}(\omega)$. We note that at higher temperature these pseudogaps disappear at the so-called pseudogap temperature $T_{1,2}^{*}[40]$.

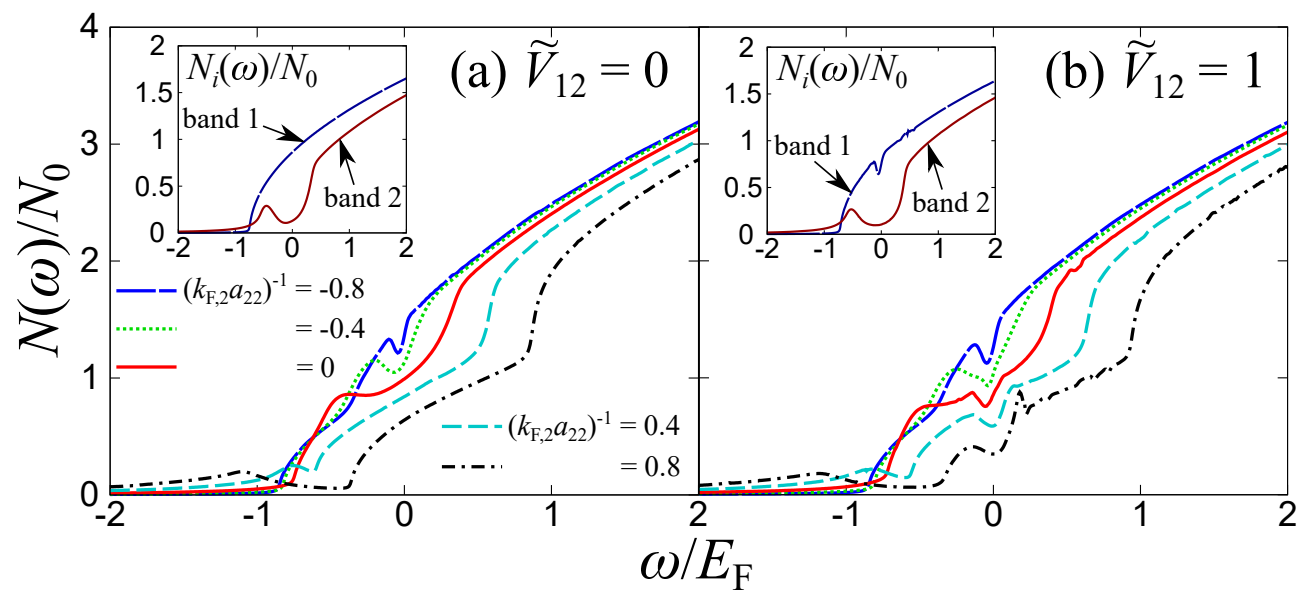

Figure 2. Total density of states $N(\omega)$ relevant for the scanning tunneling spectroscopy in the twoband BCS-BEC crossover superconductor at $T=T_{\mathrm{C}}\left[(\mathbf{a}) \tilde{V}_{12}=0,(\mathbf{b}) \tilde{V}_{12}=1\right]$. The inset shows the band-selective density of states $N_{i}(\omega)$ at $\left(k_{\mathrm{F}, 2} a_{22}\right)^{-1}=0 . N_{0}=m k_{\mathrm{F}}^{2} /\left(2 \pi^{2}\right)$ is the noninteracting density of states associated with the total number density $n$ at $T=0$.

To see the detailed structure of the pseudogaps, we discuss our results for the total spectral function $A(\mathbf{k}, \omega)$ defined as the sum of the two single-band contributions

$$
A(\mathbf{k}, \omega)=\sum_{j} A_{j}(\mathbf{k}, \omega) .
$$

This is the quantity measured by ARPES experiments. Figure 3 shows $A(\mathbf{k}, \omega)$ with $\tilde{V}_{12}=1$, where (a) $\left(k_{\mathrm{F}, 2} a_{22}\right)^{-1}=-0.4$, (b) $\left(k_{\mathrm{F}, 2} a_{22}\right)^{-1}=0$, and (c) $\left(k_{\mathrm{F}, 2} a_{22}\right)^{-1}=0.4$. Since the deep band is in the weak-coupling regime, the dispersion originating from the deep band is close to the noninteracting counterpart given by $\omega=\xi_{\mathbf{k}, 1}$. The pseudogap feature associated to the particle-hole mixing around $\omega=0$ is found to be weak in the deep band. On the other hand, the shallow band exhibits the so-called Bogoliubov-like dispersion, showing the characteristic back-bending of the dispersion for large wave-vectors, given by

$$
\omega= \pm \sqrt{\xi_{\mathbf{k}_{2}}^{2}+\Delta_{\mathrm{pg}, 2}^{2}}
$$

where $\Delta_{\mathrm{pg}, 2}$ is the pseudogap energy scale induced by strong pairing fluctuations. The backbending curve in the large wave-vector region $\left(k \gtrsim k_{\mathrm{F}}\right)$ is one of the characteristic features for the pseudogap in the angular resolved photoemission spectroscopy of ultracold Fermi gases and strongly coupled superconductors [43-46]. Since this curve is not hidden by the contribution from the deep band, it can be regarded as the signature of the pseudogap even in the present two-band system. For the case of strong intraband coupling $\left(k_{\mathrm{F}, 2} a_{22}\right)^{-1}=0.4$, the pseudogap size becomes large and the lower branch of the Bogoliubov dispersion overlaps with the deep band dispersion. This result indicates that the tightly bound molecules in the shallow band starts dominating the system even in the presence of the cold deep band due to the very strong intraband coupling in the shallow band. 


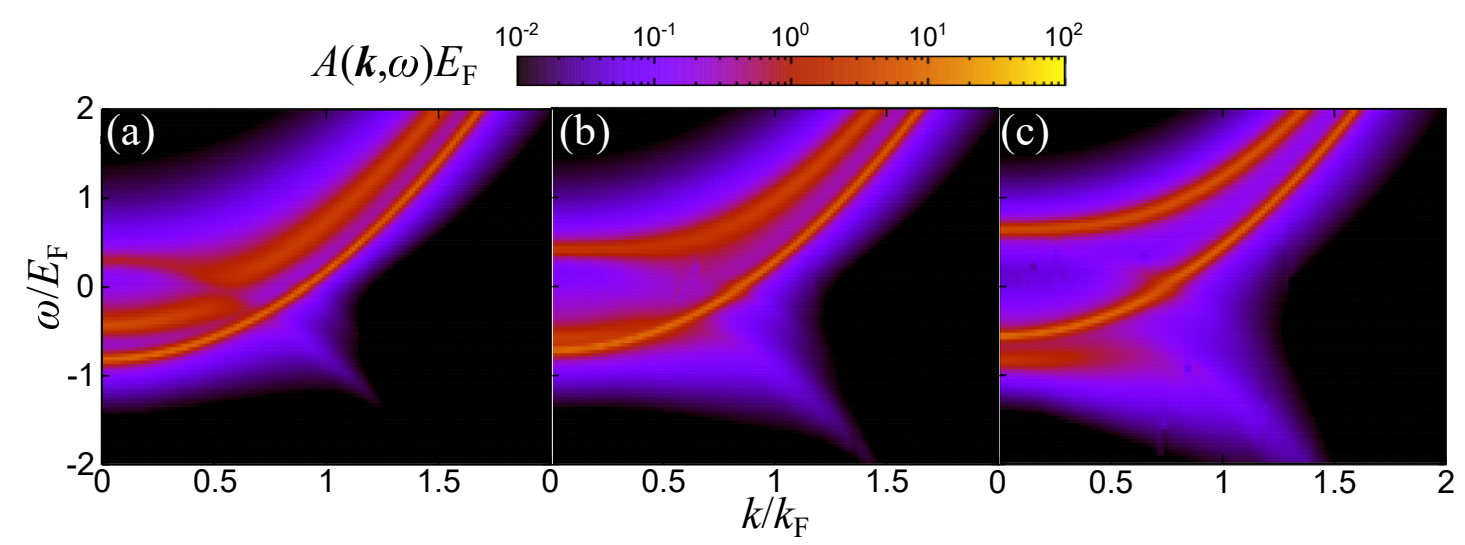

Figure 3. The total spectral function $A(\mathbf{k}, \omega)$, which is relevant for spectroscopic measurements, at $(\mathbf{a})\left(k_{\mathrm{F}, 2} a_{22}\right)=-0.4$, (b) $\left(k_{\mathrm{F}, 2} a_{22}\right)^{-1}=0$ and $(\mathbf{c})\left(k_{\mathrm{F}, 2} a_{22}\right)^{-1}=0.4$. In these panels, we use $\tilde{V}_{12}=1$.

As a final remark, we emphasize that it is necessary to solve numerically the number Equation (15) with respect to $\mu$ in a self-consistent way, to take into account the transfer of particles between the two bands, which is of key importance in the interplay between pseudogap opening and masking effect throughout the two-band BCS-BEC crossover. Moreover, the masking effect is easily overwhelmed by strong pairing fluctuations when $\tilde{V}_{12}$ is large. These nontrivial features have never been discussed in the context of the twoband BCS-BEC crossover [38-40]. Since the two-band model employed in this paper can be realized in $\mathrm{Yb}$ Fermi gases with an orbital Feshbach resonance [24] and the differential conductance corresponding to the STS will be accessible in cold atom systems [47], our prediction based on the simplified model adopted in this work will be tested experimentally in the near future.

\section{Conclusions}

We have investigated single-particle excitation spectra in a two-band superfluid/ superconductor throughout the BCS-BEC crossover and made connections with recent experiments reporting unexpected behavior of the pseudogap in multiband iron-based superconductors. Within a two-channel T-matix approach for pairing fluctuations, we have evaluated the spectral functions and the density of states for different coupling parameters, corresponding to strong pairing in the shallow band and weak paring in the deep band and different pair-exchange amplitudes. We have obtained that the pseudogap in the strongly interacting regime for the shallow band is hidden by the contribution of the weakly interacting deep band in the total density of states, which is the quantity measured by the recent STS experiments in FeSe superconductors. On the other hand, the single-particle spectral function consisting of contributions from the two bands, which is relevant to the ARPES measurement, clearly exhibits the signature of the pseudogaps, that is, the Bogoliubov back-bending dispersions. We emphasize that these nontrivial features for the pseudogaps are unique of a two-band fermionic system in which pair-fluctuations interfere in a complex manner, originating screening or amplification phenomena of superfluid/superconductor fluctuations which are absent in the single-band counterpart.

Future development of this work will aim at including a realistic band structure and effective pairing interaction suitable to describe the complex phenomenology of FeSe or other iron-based superconductors. The multiband BCS-BEC crossover and the pseudogaps will be investigated by a multichannel T-matrix approach to superconducting fluctuations in which bands of electrons and of holes and both intraband and cross-band pairing interactions will be taken into consideration.

Author Contributions: Conceptualization, H.T., P.P. and A.P.; methodology, H.T., P.P. and A.P.; software, H.T.; validation, H.T., P.P. and A.P.; writing-original draft preparation, H.T.; writingreview and editing, H.T., P.P. and A.P.; supervision, A.P. All authors have read and agreed to the published version of the manuscript. 
Funding: H.T. was supported by Grant-in-Aid for JSPS fellows (No.17J03975) and for Scientific Research from JSPS (No.18H05406).

Institutional Review Board Statement: Not applicable.

Informed Consent Statement: Not applicable.

Data Availability Statement: All data discussed in this study are available within the article.

Acknowledgments: Interesting discussions with Yuriy Yerin are acknowledged. H.T. is grateful for the hospitality of the Physics Division at the University of Camerino.

Conflicts of Interest: The authors declare no conflict of interest.

\section{References}

1. Eagles, D.M. Possible pairing without superconductivity at low carrier concentrations in bulk and thin-film superconducting semiconductor. Phys. Rev. 1969, 186, 456. [CrossRef]

2. Leggett, A.J. Diatomic molecules and Cooper pairs. In Modern Trends in the Theory of Condensed Matter; Peralski, A., Przystawa, Eds.; Springer: Berlin, Germany, 1980.

3. Nozières, P.; Schmitt-Rink, S. Bose condensation in an attractive fermion gas: From weak to strong coupling superconductivity. $J$. Low Temp. Phys. 1985, 59, 195-211. [CrossRef]

4. Sá de Melo, C.A.R.; Randeria, M.; Engelbrecht, J.R. Crossover from BCS to Bose Superconductivity: Transition Temperature and Time-Dependent Ginzburg-Landau Theory. Phys. Rev. Lett. 1993, 71, 3202. [CrossRef]

5. Regal, C.A.; Greiner, M.; Jin, D.S. Observation of Resonance Condensation of Fermionic Atom Pairs. Phys. Rev. Lett. 2004, 92, 040403. [CrossRef]

6. Zwierlein, M.W.; Stan, C.A.; Schunck, C.H.; Raupach, S.M.F.; Kerman, A.J.; Ketterle, W. Condensation of Pairs of Fermionic Atoms near a Feshbach Resonance. Phys. Rev. Lett. 2004, 92, 120403. [CrossRef]

7. Lubashevsky, Y.; Lahoud, E.; Chashka, K.; Podolsky, D.; Kanigel, A. Shallow pockets and very strong coupling superconductivity in $\mathrm{FeSe}_{x} \mathrm{Te}_{1-x}$. Nat. Phys. 2012, 8, 309-312. [CrossRef]

8. Kasahara, S.; Watashige, T.; Hanaguri, T.; Kohsaka, Y.; Yamashita, T.; Shimoyama, Y.; Mizukami, Y.; Endo, R.; Ikeda, H.; Aoyama, K.; et al. Field-induced superconducting phase of FeSe in the BCS-BEC cross-over. Proc. Natl. Acad. Sci. USA 2014, 111, 16309. [CrossRef]

9. Kasahara, S.; Yamashita, T.; Shi, A.; Kobayashi, R.; Shimoyama, Y.; Watashige, T.; Ishida, K.; Terashima, T.; Wolf, T.; Hardy, F.; et al. Giant superconducting fluctuations in the compensated semimetal FeSe at the BCS-BEC crossover. Nat. Commun. 2016, 7, 12843. [CrossRef] [PubMed]

10. Rinott, S.; Chashka, K.B.; Ribak, A.; Rienks Emile, D.L.; Taleb-Ibrahimi, A.; Le Fevre, P.; Bertran, F.; Randeria, M.; Kanigel, A. Tuning across the BCS-BEC crossover in the multiband superconductor $\mathrm{Fe}_{1+y} \mathrm{Se}_{x} \mathrm{Te}_{1-x}$ : An angle-resolved photoemission study. Sci. Adv. 2017, 3, e1602372. [CrossRef] [PubMed]

11. Hashimoto, T.; Ota, Y.; Tsuzuki, A.; Nagashima, T.; Fukushima, A.; Kasahara, S.; Matsuda, Y.; Matsuura, K.; Mizukami, Y.; Shibauchi, T.; et al. Bose-Einstein condensation superconductivity induced by disappearance of the nematic state. Sci. Adv. 2020, 6, eabb9052. [CrossRef] [PubMed]

12. Valletta, A.; Bianconi, A.; Perali, A.; Saini, N.L. Electronic and superconducting properties of a superlattice of quantum stripes at the atomic limit. Z. Phys. B Condens. Matter 1997, 104, 707-713. [CrossRef]

13. Iskin, M.; Sá de Melo, C.A.R. Two-band superfluidity from the BCS to the BEC limit. Phys. Rev. B 2006, 74, 144517. [CrossRef]

14. Innocenti, D.; Poccia, N.; Ricci, A.; Valletta, A.; Caprara, S.; Perali, A.; Bianconi, A. Resonant and crossover phenomena in a multiband superconductor: Tuning the chemical potential near a band edge. Phys. Rev. B 2010, 82, 184528. [CrossRef]

15. Guidini, A.; Perali, A. Band-edge BCS-BEC crossover in a two-band superconductor: physical properties and detection parameters. Supercond. Sci. Technol. 2014, 27, 124002. [CrossRef]

16. He, L.; Hu, H.; Liu, X.-J. Two-band description of resonant supefluidity in atomic Fermi gases. Phys. Rev. A 2015, 91, 023622. [CrossRef]

17. He, L.; Wang, J.; Peng, S.-G.; Liu, X.-J.; Hu, H. Strongly correlated Fermi superfluid near an orbital Feshbach resonance: Stability, equation of state, and Leggett mode. Phys. Rev. A 2016, 94, 043624. [CrossRef]

18. Chubukov, A.V.; Eremin, I.; Efremov, D.V. Superconductivity versus bound-state formation in a two-band superconductor with small Fermi energy: Applications to Fe pnictides/chalcogenides and doped SrTiO . Phys. Rev. B 2016, 93, 174516. [CrossRef]

19. Wolf, S.; Vagov, A.; Shanenko, A.A.; Axt, V.M.; Perali, A.; Albino Aguiar, J. BCS-BEC crossover induced by a shallow band: Pushing standard superconductivity types apart. Phys. Rev. B 2017, 95, 094521. [CrossRef]

20. Mondal, S.; Inotani, D.; Ohashi, Y. Single-particle Excitations and Strong-Coupling Effects in the BCS-BEC Crossover Regime of a Rare-Earth Fermi Gas with an Orbital Feshbach Resonance. J. Phys. Soc. Jpn. 2018, 87, 084302. [CrossRef]

21. Yerin, Y.; Tajima, H.; Pieri, P.; Perali, A. Coexistence of giant Cooper pairs with a bosonic condensate and anomalous behavior of energy gaps in the BCS-BEC crossover of a two band superfluid Fermi gas. Phys. Rev. B 2019, 100, 104528. [CrossRef]

22. Aoki, H. Theoretical Possibilities for Flat Band Superconductivity. J. Supercond. Nov. Magn. 2020, 33, 2341-2346. [CrossRef] 
23. Suhl, H.; Matthias, B.T.; Walker, L.R. Bardeen-Cooper-Schrieffer Theory of Superconductivity in the Case of Overlapping Bands. Phys. Rev. Lett. 1959, 3, 552. [CrossRef]

24. Zhang, R.; Cheng, Y.; Zhai, H.; Zhang, P. Orbital Feshbach Resonance in Alkali-Earth Atoms. Phys. Rev. Lett. 2015, 115, 135301. [CrossRef]

25. Pagano, G.; Mancini, M.; Cappellini, G.; Livi, L.; Sias, C.; Catani, J.; Inguscio, M.; Fallani, L. Strongly Interacting Gas of Two-Electron Fermions at an Orbital Feshbach Reonance. Phys. Rev. Lett. 2015, 115, 265301. [CrossRef]

26. Höfer, M.; Riegger, L.; Scazza, F.; Hofrichter, C.; Fernandes, D.R.; Parish, M.M.; Levinsen, J.; Bloch, I.; Folling, S. Observation of an Orbital Interaction-Induced Feshbach Resonance in ${ }^{173}$ Yb. Phys. Rev. Lett. 2015, 115, 265302. [CrossRef]

27. Mueller, E.J. Review of pseudogaps in strongly interacting Fermi gases. Rep. Prog, Phys. 2017, 80, 104401. [CrossRef] [PubMed]

28. Calvanese Strinati, G.; Pieri, P.; Röpke, G.; Schuck, P.; Urban, M. The BCS-BEC crossover: From ultra-cold Fermi gases to nuclear systems. Phys. Rep. 2018, 738, 1. [CrossRef]

29. Ohashi, Y.; Tajima, H.; van Wyk, P. BCS-BEC crossover in cold atomic and in nuclear systems. Prog. Part. Nucl. Phys. 2020, 111, 103739. [CrossRef]

30. Gati, E.; Böhmer, A.E.; Bud'ko, S.L.; Canfield, P.C. Bulk superconductivity and role of fluctuations in the iron based superconductor FeSe at high pressures. Phys. Rev. Lett. 2019, 123, 167002. [CrossRef]

31. Seo, Y.I.; Choi, W.J.; Kimura, S.; Kwon, Y.S. Evidence for a preformed Cooper pair model in the pseudogap spectra of a $\mathrm{Ca}_{10}\left(\mathrm{Pt}_{4} \mathrm{As}_{8}\right)\left(\mathrm{Fe}_{2} \mathrm{As}_{2}\right)_{5}$ single crystal with a nodal superconducting gap. Sci. Rep. 2019, 9, 3987. [CrossRef] [PubMed]

32. Kang, B.L.; Shi, M.Z.; Li, S.J.; Wang, H.H.; Zhang, Q.; Zhao, D.; Li, J.; Song, D.W.; Zheng, L.X.; Nie, L.P.; et al. Preformed Cooper Pairs in Layered FeSe-Based Superconductors. Phys. Rev. Lett. 2020, 125, 097003. [CrossRef]

33. Solovjov, A.L.; Petrenko, E.V.; Omelchenko, L.V.; Nazarova, E.; Buchkov, K.; Rogacki, K. Fluctuating Cooper pairs in FeSe at temperatures exceeding double $T_{\mathrm{c}}$. arXiv 2020, arXiv:2010.12319.

34. Hanaguri, T.; Kasahara, S.; Böker, J.; Eremin, I.; Shibauchi, T.; Matsuda, Y. Quantum Vortex Core and Missing Pseudogap in the Multiband BCS-BEC Crossover Superconductor FeSe. Phys. Rev. Lett. 2019, 122, 077001. [CrossRef]

35. Takahashi, H.; Nabeshima, F.; Ogawa, R.; Ohmichi, E.; Ohta, H.; Maeda, A. Superconducting fluctuations in FeSe investigated by precise torque magnetometry. Phys. Rev. B 2019, 99, 060503(R). [CrossRef]

36. Salasnich, L.; Shanenko, A.A.; Vagov, A.; Albino Aguiar, J.; Perali, A. Screening of pair fluctuations in superconductors with coupled shallow and deep bands: A route to higher-temperature superconductivity. Phys. Rev. B 2019, 100, 064510. [CrossRef]

37. Saraiva, T.T.; Cavalcanti, P.J.F.; Vagov, A.; Vasenko, A.S.; Perali, A.; Dell'Anna, L.; Shanenko, A.A. Multiband Material with a Quasi-1D Band as a Robust High-Temperature Superconductor. Phys. Rev. Lett. 2020, 125, 217003. [CrossRef] [PubMed]

38. Tajima, H.; Yerin, Y.; Perali, A.; Pieri, P. Enhanced critical temperature, pairing fluctuation effects, and BCS-BEC crossover in a two band Fermi gas. Phys. Rev. B 2019, 99, 180503(R). [CrossRef]

39. Tajima, H.; Pieri, P.; Perali, A. BCS-BEC Crossover and Pairing Fluctuations in a Two Band Superfluid/Superconductors: A T Matrix Approach. Condens. Matter 2020, 5, 10. [CrossRef]

40. Tajima, H.; Yerin, Y.; Pieri, P.; Perali, A. Mechanism of screening or enhancing the pseudogapp throughout the two-band Bardeen-Cooper-Schrieffer to Bose-Einstein condensate crossover. Phys. Rev. B 2020, 102, 220504(R). [CrossRef]

41. Fischer, Ø.; Kugler, M.; Maggio-Aprile, I.; Berthod, C.; Renner, C. Scanning tunneling spectroscopy of high-temperature superconductors. Rev. Mod. Phys. 2007, 79, 353. [CrossRef]

42. Vargas-Paredes, A.A.; Shanenko, A.A.; Vagov, A.; Milošević, M.V.; Perali, A. Crossband versus intraband pairing in superconductors: Signatures and consequences of the interplay. Phys. Rev. B 2020,101, 094516. [CrossRef]

43. Perali, A.; Pieri, P.; Strinati, G.C.; Castellani, C. Pseudogap and spectral function from superconducting fluctuations to the bosonic limit. Phys. Rev. B 2002, 66, 024510. [CrossRef]

44. Gaebler, J.P.; Stewart, J.T.; Drake, T.E.; Jin, D.S.; Perali, A.; Pieri, P.; Strinati, G.C. Observation of pseudogap behaviour in a strongly interacting Fermi gas. Nat. Phys. 2010, 6, 569. [CrossRef]

45. Perali, A.; Palestini, F.; Pieri, P.; Strinati, G.C.; Stewart, J.T.; Gaebler, J.P.; Drake, T.E.; Jin, D.S. Evolution of the Normal State of a Strongly Interacting Fermi Gas from a Pseudogap Phase to a Molecular Bose Gas. Phys. Rev. Lett. 2011, 106, 060402. [CrossRef] [PubMed]

46. Palestini, F.; Perali, A.; Pieri, P.; Strinati, G.C. Dispersions, weights, and widths of the single-particle spectral function in the normal phase of a Fermi gas. Phys. Rev. B 2012, 85, 024517. [CrossRef]

47. Del Pace, G.; Kwon, W.J.; Zaccanti, M.; Roati, G.; Scazza, F. Tunneling transport of unitary fermions across the superfluid transition. arXiv 2020, arXiv:2010.00582. 